\title{
MedienPädagogik
}

Zeitschrift für Theorie und Praxis der Medienbildung

\section{Editorial: Methodologische Forschungsansätze}

\author{
Heinz Moser
}

Das vorliegende Heft der Zeitschrift MedienPädagogik umfasst vor allem Beiträge, die aus der Sicht der qualitativen Forschung geschrieben wurden. Das war nicht unbedingt die Absicht des Herausgebers. Doch alle Beiträge, die uns auf Grund unseres «calls for paper» erreichten, bezogen sich auf Thematiken im Rahmen der Begründung und Diskussion qualitativer Forschungsverfahren.

Wir sehen darin einen Ausdruck der Tatsache, dass in den letzten Jahren die Methoden einer qualitative Forschungsmethodologie zunehmend salonfähig geworden sind. Gerade wenn man Medienereignisse unter dem Gesichtspunkt aktiver Rezipienten betrachtet, die nicht einfach Opfer von externen Reizen sind, dann bietet es sich an, diese Interaktivität mit qualitativer Mitteln zu untersuchen. Möglich sind z.B. teilnehmende Beobachtung, Tiefen- oder Gruppeninterviews. All diesen Verfahren ist gemeinsam, dass die Forschenden in eine intensive Beziehung zu ihrem Gegenstand eingetreten, wie sie über die objektivierenden Verfahren der klassischen Empirie kaum möglich ist.

Ein zweites Merkmal qualitativer Forschung ist es, dass es in solchen Untersuchungen oft weniger um die Überprüfung von Theorien geht, sondern um deren Entwicklung. Kleine Gruppen eignen sich besonders, um im Kontakt mit den Daten theoriegenerierende Arbeit zu leisten. Das Konzept der «grounded theory» betont z.B. explizit, dass damit ein Weg verbunden sei, um Theorien aus den untersuchten Fällen heraus zu entwickeln.

Was drittens besonders zur einer medienpädagogischen Zeitschrift passt, ist der Umstand, dass in dieser Nummer auch Möglichkeiten eines Einbezug von visuellen Verfahren in qualitative Forschungsaktivitäten diskutiert wird. Damit wird signalisiert, dass Medien nicht nur Gegenstand dieser Zeitschrift sind, sondern - wie im Rahmen von Videoanalysen - auch ein Instrument in der Hand von Forscher/innen sein können, um den «Untersuchungsgegenstand» nicht allein über schriftliche Texte (Interviews, Fragebögen) zu repräsentieren.

Zum Schluss eine Bemerkung in eigener Sache: Die nachfolgenden Beiträge sind insbesonders auch als Diskussionsbeiträge zu verstehen. Gerade in einem OnlineJournal ist es besser möglich, Kommentare, Reaktionen und Diskussionsbeiträge von Leser/innen einzubeziehen. Aus diesem Grunde besteht in dieser Zeitschrift auch ein Forum, in welchem die Thesen und Aussagen der Autor/innen weiterdiskutiert werden können. Wir würden uns freuen, wenn Sie diese Möglichkeit nutzen und Ihre Kommentare und Stellungnahmen dort publizieren würden.

Moser, Heinz. 2001. «Editorial: Methodologische Forschungsansätze». MedienPädagogik 3, (Februar), i. https://doi.org/10.21240/ mpaed/03/2001.02.19.X. 